\title{
Motion Induced Colours Fusion
}

\author{
Ahmad Yousef ${ }^{1}$ \\ ${ }^{1}$ School of Computational Science and Engineering, McMaster University, Hamilton, Ontario, Canada \\ *Correspondence: mohamas2@mcmaster.ca
}

\begin{abstract}
This article provides evidence that motion has the ability to stably fuse two different colored 'spatially separated' stimuli without impairments against the shape of those separated stimuli. Based on our observations, dilated pupils promote colours fusion; and the optimal fusion happens when the stimuli are moving in opposite direction of the actual physical motion, namely, when there is illusory motion reversal. The previous observations suggested that retinal peripheries have main contributions on the present illusion which we called; motion induced colours fusion 'MICF' illusion.

Introduction

Both Helmholtz's trichromatic, and Hering's opponent process theories are widely accepted by the scientific community, however, it's believed that trichromacy occurs at the photoreceptors' level, but the opponent processes emerges at the retinal ganglion cells' level and in the brain, including but not limited to, several cortical areas. In opponent process theory, the system works based on the opposing color effect of red-green, blue-yellow, and light-dark. However, it is the visual neural processes of various types of photoreceptors that are opposed.
\end{abstract} Since Hering's theory is a neuropsychological structure that describes a wide spectrum of behavioral dynamics, including color vision; it therefore might bring explaining to our present illusion. Needless to mention, the brain is fed through optic chiasma, a neurological entity that sends visual information to the lateral geniculate nucleus from retinal ganglion cells. The lateral geniculate nucleus is divided into three zones 'laminaes'; M- laminae, P- laminae, and koniocellular laminae. $\mathrm{M}$ - and $\mathrm{P}$-cells are the main building blocks of $\mathrm{P}$ - laminae, and $\mathrm{M}$ - laminae respectively. These cells receive relatively balanced input from both $L$ and $M$ retinal cones from the entire retina, not just from the fovea. Koniocellular laminae, in the other hand, gathers neurophysiological signals from the small bistratified ganglion cells, see reference 3 , and 4 .

Afterwards, striate cortex of $\mathrm{V} 1$ will be synapsed, a region that assumes to achieve simple three-color segregation. Important to mention, many cells in V1 respond to some parts of the light spectrum better than others in relatively bright conditions; in dim conditions, however, those cells might respond to the entire spectrum. Noticeably, in bright environments, pupil constricted, and the anatomical lens mostly flattered; however, in dim environments, the pupil dilated, the anatomical lens be mostly rounded, see reference, 5 . The aforementioned observations might suggest that $\mathrm{V} 1$ might be in consistence with the two conscious brains hypothesis, see reference 6 . In another word, V1 might have two distinct areas, namely, a central area which is neurological connected to the fovea and has decent abilities to distinguish between different colors; and a peripheral area connected to the retinal peripheries which has much weaker color segregation, and thus the segregation process might require more neural processes in further cortical areas. Important to emphasize, when we say V1 might have central and peripheral areas; this doesn't mean these two distinct areas have to be geometrically in the center and the peripheries of $\mathrm{V} 1$, but they are connected to the fovea and the retinal peripheries respectively. Due to the aforementioned issue, "double-opponent" cells were suggested, see reference 7; these cells that can be found in blobs regions in V1 area, see reference 8 . In retinex theory, doubleopponent cells are assumed to be outstanding candidates for color constancy neural machinery, see reference 9 . Those cells forwarded their color information to V2 cells; cells that are optimally colored tuned. Extended V4, a large cortical region that includes $V 4$ area and many other areas, will be afterwards synapsed by V2 neurons, see reference 10, and 11. Finally and most importantly, to approach neurophysiological understandings to the current illusion; we have to grasp information about the cues' integrations. Anatomical and functional brain imaging studies have demonstrated that neurons in extended V4 area could synapse the inferior temporal lobe (IT). The aforementioned cortical area had been assumed to integrate color information with shape and form, see reference 12 . Important to mention, both color and motion defined shapes trigger higher $\mathrm{FMRI}$ activities gainst the actual motion in the lateral occipital complex, see reference 13; and that shapes defined by both color and motion simultaneously stimulate the anterior ventral margins of this area more strongly than shapes defined by either cue alone. Because we had noticed outstanding integration between color, motion and shape in our illusory stimuli, see reference 14; and based on the previous studies, IT cortex, lateral occipital complex are the best candidates to investigate the neural substrates of the 'MICF' illusion. IT cortex, and lateral occipital complex might be therefore held the neural terminals that feeds the human visual awareness, see reference 15 . Important to emphasize, since our twelve subjects had noticed that the direction of motion of the little circles is opposite to the actual 'physical' direction and the speed itself is much slower, in another word, illusory motion reversal; the retinal peripheries and its corresponding subcortical and cortical networks should be integrated in the imaging studies, see reference, 16.

\section{Materials \& Methods}

Twelve human subjects, with dilated pupils, were recruited to participate in this study. Each stimulus subtends 12.4 DVA in length, and 1.72 DVA in width, and each little circle subtends 0.57 DVA. Each human subject has to undergo into two major trials; the first trail consists of three different colors combinations for little circles; red-green, red-blue, and green-blue, and to be 
counterbalanced; an example of the visual awareness of the stimulus in motion is exhibited in page 1 central image, and in reference 13 . In motion, human subjects have reported that they hadn't see pure colors but no impairments against the shape of the circles; thus, they were requested, in the second trial, to report the strength of these motion-induced colors compared with frame of references, namely, pure yellow, magenta, and cyan. Important to mention, the impurity of the yellow color in the page 1 central image is $10 \%$ additional red


or green to $90 \%$ pure yellow. Important to emphasize, we had requested from the human subjects to compare the motioninduced colors with pure colors as frame of references, because they have reported colors such as greenish yellow, reddish magenta, greenish blue, etc. It was noticed among all of the human subjects that the fusion takes a few seconds ( 1 - 4 seconds) to be started until the pupil and the lens are being adapted. Astonishingly, despite the stimuli move at speed of 14.4 degrees/sec, once optimal colour fusion occurs which had been connected to illusory motion reversal, impairments against the shape of the little circles were disappeared. Some human subjects had reported difficultly to fuse green - blue, and red - blue; but no difficulties at all for red-green. The blue element therefore might trigger some dynamics in the human visual system that reduce this illusory fusion; blue color is known to be a very dark color, and since it resides against black background; the local contrast will be extremely low. Since reducing the contrast constricts the pupil size, see reference 17; we therefore had requested from the subjects to attend peripherally to feed the retinal peripheries with more intense light rays, see page 1 central figure. After the retest, all of the human subjects were able to see optimal colour fusion with almost no impairments against the shape of the circles. The aforementioned observations brought evidence supporting our hypothesis that states; the retinal peripheries are the main contributors in creating the ' $M I C F$ ' illusion!

Data Collection

The behavioral data were collected manually, and then averaged among the human subjects to estimate the statistical values, namely, the average and the standard error of the mean of the strength of the fused colors compared to frames of references. Results and Conclusion

As shown in the up-central infographic, the fusion strength is significantly high. It was astounding to notice that the arrows are moving in the actual physical direction; but the little circles are moving in the opposite direction with no impairments against their shapes despite the high speed; as if the little circles had constructed a background that is completely detached from the moving arrows. These observations might suggest that the human visual system is dealing with the stimulus in simultaneously two different ways, namely, the detachment might be possibly produced by summing up the visual awareness of the central plus the peripheral conscious brains. Observations that may tell us that there might be exceptional entanglement communications between the different geometrical shapes, and the different areas in the retina; for fulsome comprehension, see reference 15 . Essential Notification Readers should have direct access to the stimuli, see reference 14. Advisably, download the videos for the best quality. Wait for a few seconds to allow adaptation to the pupil and the lens; the illusion afterwards should appear. For rapid validation process, have the test with dilated pupil, watch the stimulus peripherally as shown page 1 central figure. and watch the videos in moderate environment; namely, fifty percent of the monitor's max. brightness. Caffeine intake also dilate the pupil, in case of pinpoint pupils, see reference 18. You may stop the video at any time for several confirmations! Scholars with brain imaging facilitations are welcome to collaborate in challenging investigations, namely, to achieve critical analyses of the spatiotemporal properties of the IT cortex, and lateral occipital complex for possibly better understanding of the neural substrates of this motion-induced color fusion 'MICF' illusion. Enquiries may be sent to the author.

Transactional References

[1] Thomas Young, (1802). Bakerian Lecture: On the Theory of Light and Colours. Phil. Trans. R. Soc. Lond. 92:12-48. doi: 10.1098/rstl. 1802.0004

[2] Ewald Hering, (1878) "Opponent-process Theory." Expanded by Richard Solomon.

[3] R.W. Rodieck, (1998) "The First Steps in Seeing". Sinauer

Associates, Inc., Sunderland, Massachusetts.

[4]Hendry, and Reid, (2000)"The Koniocellular Pathway in Primate Vision". Annual Reviews Neuroscience.

[5] Yousef, A. 2019. "Linking Dynamics Between Pupil and Lens." engrXiv. June 26. doi:10.31224/osf.io/9c3vk.

[6] Yousef, A. 2019. "Two Distinct Fusiform Face Areas." PsyArXiv. doi:10.31234/osf.io/a8gzv.

[7] Conway, (2001). "Spatial structure of cone inputs to color cells in alert macaque primary visual cortex $(\mathrm{N}-1)$ ". Journal of Neuroscience. [8] J. Dowling (2001). Neurons and Networks: An Introduction to Behavioral Neuroscience. Harvard University Press.

[9] McCann, (1993). "Edwin H. Land's Essays." Society for Imaging Science and Technology.

[10] Conway, Moeller, and Tsao (2007). "Specialized color modules in macaque extrastriate cortex". Neuron.

[11] Conway, and Tsao (2009). "Color-tuned neurons are spatially clustered according to color preference within alert macaque posterior inferior temporal cortex". PNAS.

[12] Komatsu, and Ideura (1993) "Relationships between color, shape, and pattern selectivities of neurons in the inferior temporal cortex of the monkey." J. Neurophysiology.

[13] M. Self, and S. Zeki (2005). "The Integration of Colour and Motion by the Human Visual Brain". Cerebral Cortex.

[14] You may find the stimuli in the following link: https://drive.google.com/drive/folders/1S8vFItQepWIAoVIzOh5TCXL R-uOag9VH

[15] Yousef, A. 2019. "Consciousness Might Be Localized in Extra Physical Dimensions." PsyArXiv. doi:10.31234/osf.io/angc8.

[16] Yousef, A. 2019. "Retinal Peripheries Generate Illusory Motion Reversals." PsyArXiv. doi:10.31234/osf.io/gfmpe.

[17] Wang, etal (2014) Modulation of stimulus contrast on the human pupil orienting response, in European Journal of Neuroscience, [18] Abokyi, etal.,(2017). Caffeine intake is associated with pupil dilation and enhanced accommodation. Eye (Lond) Journal. 